\title{
Face Detection Using Haar Cascades Classifier
}

\author{
Harleen Kaur*, Arisha Mirza \\ \{*harleen.unu@gmail.com\} \\ Department of Computer Sciences and Engineering, School of Engineering Sciences and Technology, \\ Jamia Hamdard, New Delhi, India
}

\begin{abstract}
In the last several years, face detection has been listed as one of the most engaging field in research. Face detection algorithms is used for detection of frontal human faces. Face detection find use is many applications such as face tracking, faces analysis and face recognition. In this paper, we are going to discuss face detection using a haar cascade classifier and OpenCV. In this study we would be focusing on some of the face detection technology in use.
\end{abstract}

Keywords: Face detection, Haar cascades classifier, OpenCV, Scope of face detection, Feed-forward neural networks, deep learning.

\section{Introduction}

Face detection concerns about and focuses on locating a face in an image. It might seem to you very easy but there are many limitations in the same such as single or multiple faces, pose, image rotation etc. We will start this study with a brief introduction in face detection technology and its applications and use. Face Detection is seen as an AI based computer technology that can be used to identify focuses and locate the presence of human faces in digital photos, videos and other media [1].

Machine learning is said to be a part or subset of artificial intelligence that includes some techniques like data analysis and pattern recognition. Algorithms are designed in such a way so that a computer can learn from a given data set or raw data on its own and not getting involved in the interesting facts and figures about the data. Our primary focus will be on how to make use of these algorithms in the best way possible. The use and applications of these algorithms are making their use in almost every field and industry in today's time [2].

Many of the algorithms and techniques are used in face detection but some of them had their own limitations and drawbacks. Most of the techniques appointed for this work in other applications had their own drawbacks too, but we have to focus on how to minimize these drawbacks and limitations and maximize its performance and accuracy. The methodology for this includes techniques like facial feature extraction, normalization, image reduction and classification.

\section{Facial features extraction}


Features, such as eyes, nose, and mouth are very important in various applications like face recognition, facial expression detection, face tracking, lip-reading, etc. Hence, after detecting the face region from an image we should extract various features from the face, and then we verify them for our recognition purpose [9-13]. Extraction property of different features can be coded either manually or by using some procedure from a set of data [11]. There are five types of feature extraction methods: Generic methods; Gabor wavelets; Feature template-based methods that are used to detect various facial features like eyes, nose, and mouth; Colour segmentation based methods; Appearance-based methods that can manage changes in illumination conditions, shape, pose and reflectance.

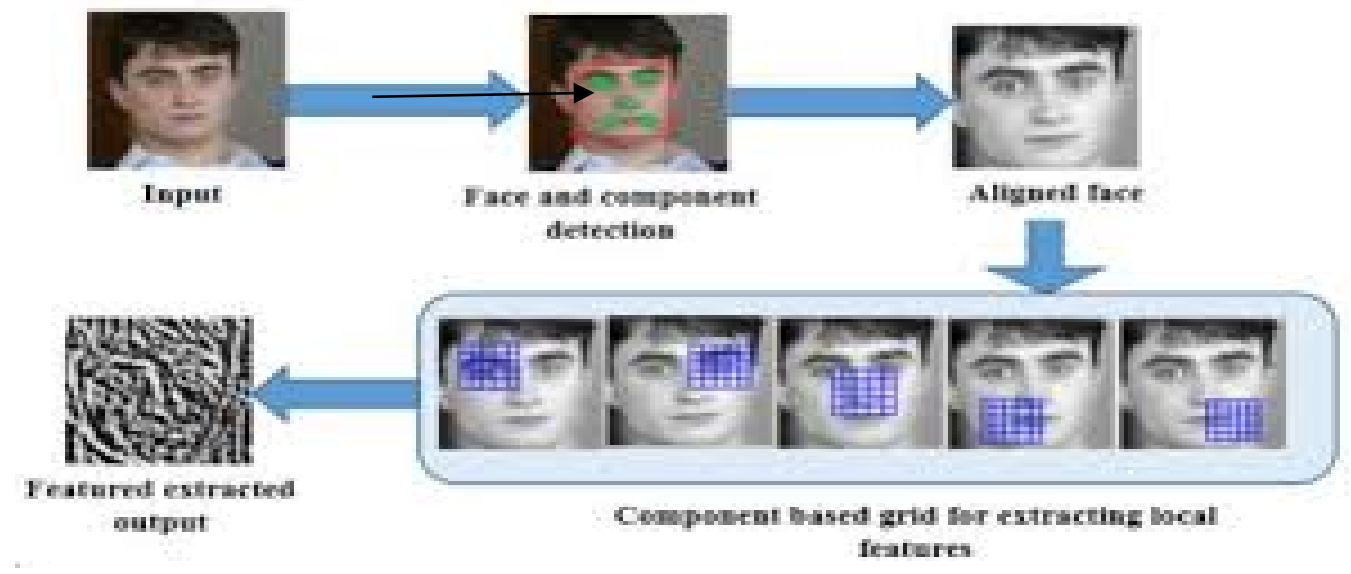

Fig. 1. Facial features detection

\section{Literature survey}

The Facial detection system is one of the favourite research areas. The work defined by the earlier researchers on facial detection is discussed in this section. There have been a lot of studies done about face detection techniques and algorithms. We will discuss a few popular methods for face detection.

ISMAIL and MD. SABRI, 2009 [3] has presented work on some used techniques for face recognition and its detection. The Author defines the most successful existing algorithms and techniques which can be used for face recognition technology. This review is written by searching past and current studies done by different researchers associated with a similar subject. Five different algorithms have been chosen based on the most widely used criteria.

Ebenezer Owusu et al., 2018 [4] has shown the face detection method using the haar feature extraction and classification by MFNN. His motive for this study was improving its accuracy. The salient facial expressions for this study are extracted through haar techniques.

Mehta et al., 2018 [2] have presented work on face detection technology using deep learning. In his study, he extended the working of multi-view face detection using Convolution Neural Networks used by Farfade et al. thus providing an essential face detecting system. 
Now during this paper, we discussed the new technologies in face detection. And therefore the future scope of face detection technology. We develop a real- time face detection project to get information about the working of a face detection system.

\section{Methodology}

The face detection technology is divided into two steps, of which the first step of this technology is the classification task that take some random picture or media as input and gives an output in a form of a binary value of yes or no, showing us whether there are any faces present in the image or not. The second step is known as a face localization task that includes an image as input and gives the location of any face within that image as an output in a bounding box with dimensions as width and height. In this study we will use OpenCV for our face detection technology.

\subsection{OpenCV}

OpenCV, an abbreviation for open source computer vision library, it is an open source library used for computer vision and also a machine learning library that finds applications in many fields including face detection. OpenCV was designed to produce a standard base to be used in computer vision techniques and applications [5]. To get started with this face detection technology, there are some modules that are to be imported and that include the following.
1. Cv2
2. Numpy
3. Sys

\subsection{Haar Cascades Classifier}

Haar cascade classifier is one of the efficient ways for object detection method and it was proposed in a paper by Paul Viola and Michael Jones, "Rapid Object Detection uses a Boosted Cascade of straightforward Features". It can be seen as a machine learning approach where we have a cascade function and is trained from lot of images as inputs [6-8]. Then it can be used to observe objects in different media and images.

In starting, the formula needs an excellent amount of images of faces and without faces to train the classifier. The haar features used are shown in the image below fig 2 .

The face detection is done using these subsequent classifiers:

Haar cascade_eye

Haar cascade_frontalface_default. 

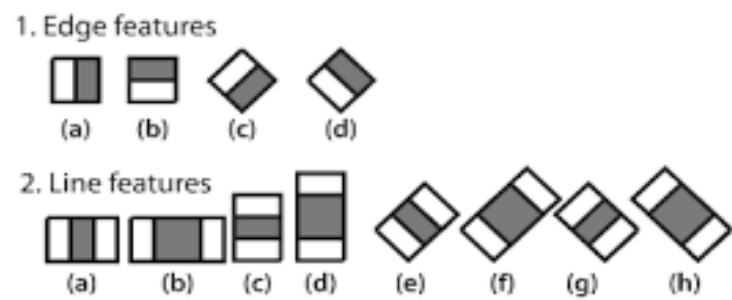

3. Center-surround features

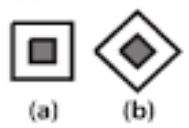

Fig. 2. Haar cascade classifier working

\section{Results and Discussions}

First, we want to put in an OpenCV package to be imported for python [6]. This can be done by downloading the following package from the python site or by using the pip install command in case you are working on Linux. For this we will need two cascade files and they are haarcascade_frontalface_default.xml and haarcascade_eye.xml. The implementation of code is done in python and we also need a working webcam to capture images or videos for testing the working model. After the model is executed successfully or working it will be able to recognize faces in still images, videos, webcam capture etc. The system first detects the faces from an image that is given as an input, which in return gives an intermediate output. Figure-4 shows the detection of multiple faces irrespective of all the different faces. Figure-5 shows the detection of the partially hidden faces and figure- 6 shows the detection of faces which are the blurred.

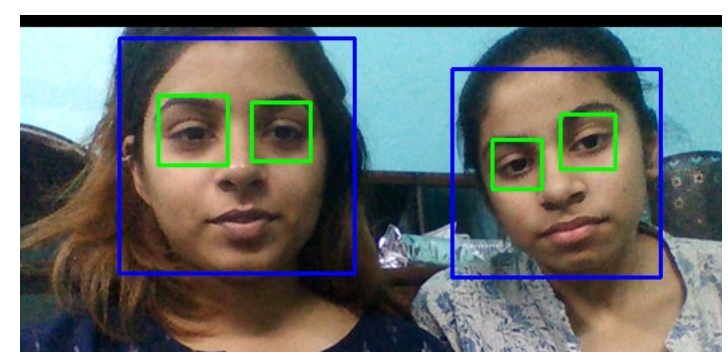

Fig. 3. Output of the recognized faces 


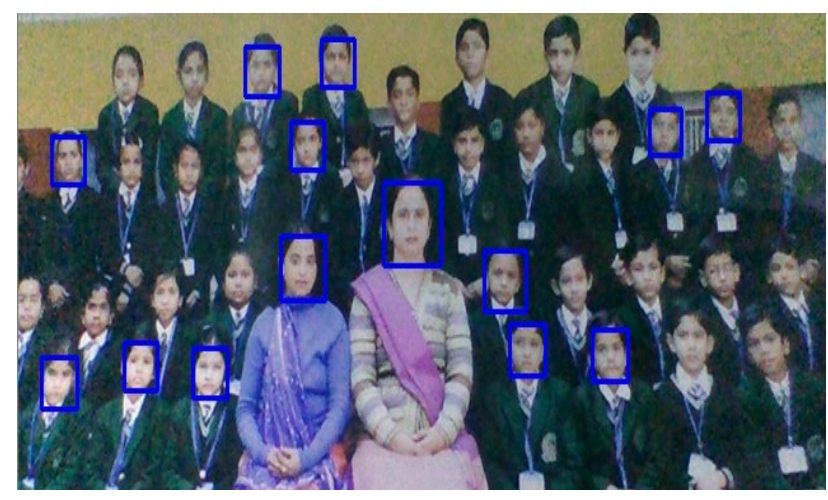

Fig. 4. Detection of Multiple faces

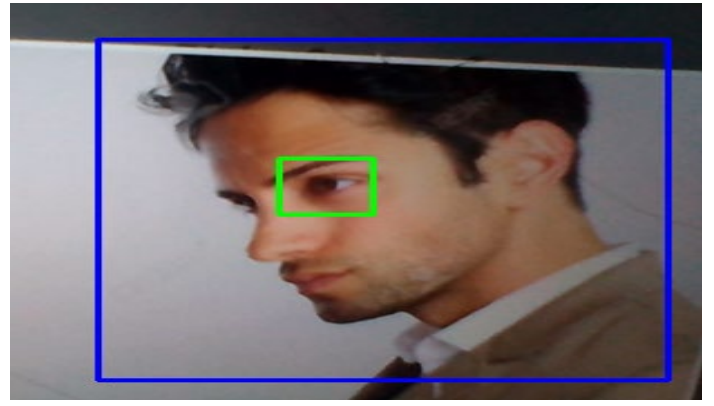

Fig. 5. Face slightly hidden

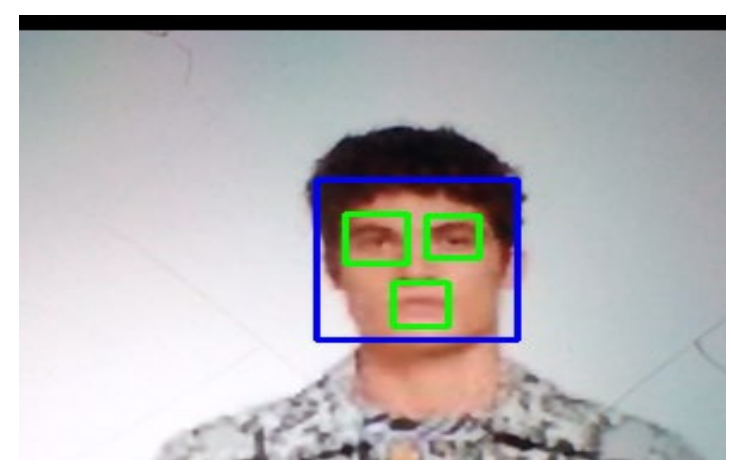

Fig. 6. Blurred image

\section{Conclusion}


In this study, we covered and studied in detail about face detection technique using haar cascades classifier and OpenCV to get the desired output. Using OpenCV library, haar cascade classifier was able to perform successful face detection with high accuracy and efficiency. We also used the OpenCV package to extract some of the features of the face to compare them. Also, we discussed some popular face detection methods. Further, we discussed the scope of face detection in the future and some of its applications. At last, we conclude that the future of facial detection technology is bright Security and surveillance is the major segments that will be deeply influenced. Other areas that are now welcoming it are private industries, public buildings, and schools.

\section{References}

[1] sightcorp, "face detection- what is face detection?," 2019. [Online]. Available: https://sightcorp.com/knowledge-base/face-detection/.

[2] Mehta J, Ramnani E, Singh S.: Face Detection and Tagging Using Deep Learning. In 2nd International Conference on Computer, Communication, and Signal Processing: Special Focus on Technology and Innovation for Smart Environment, ICCCSP 2018. https://doi.org/10.1109/ICCCSP.2018.8452853

[3] Ismail, Nurulhuda, Idayu, Mas, Md Sabri, Mas Idayu. : Review of existing algorithms for face detection and recognition.. pp. 1-39 (2009).

[4] Owusu E, Abdulai J-D, Zhan Y.: Face detection based on multilayer feed-forward neural network and Haar features. Softw Pract Exper. pp.1-10 (2018). https://doi.org/10.1002/spe.2646.

[5] opencv team, "opencv.org," 2019. [Online]. Available: https://opencv.org/about/.

[6] openCV.org, "openCV : cascade classifier," 2020.

[Online].Available:https://docs.opencv.org/3.4/db/d28/tutorial_cascade_classifier.html.

[7] Sharma, Manik \& Anuradha, J. \& Manne, H \& Kashyap, G.: Facial detection using deep learning. IOP Conference Series: Materials Science and Engineering. pp. 1-9 (2017).

[8] Viola, Paul \& Jones, Michael. : Rapid Object Detection using a Boosted Cascade of Simple Features. IEEE Conf Comput Vis Pattern Recognit. pp. 1-9 (2001).

[9] S, Suchitra.: Face Image Retrieval of Efficient Sparse Code words and Multiple Attribute in Binning Image. Brazilian Archives of Biology and Technology. pp. 1-15 (2017).

[10] O. Kharkovyna, "Facial Recognition And AI: Latest Developments And Future Directions," 2017. [Online]. Available: https://becominghuman.ai/facial-recognition-and-ai-latest-developmentsand-futuredirections-39d22201d $88 \mathrm{~b}$.

[11] S. Tiwari, "Face Recognition with Python, in Under 25 Lines of Code," 2012. [Online]. Available: https://realpython.com/face-recognition-withpython/. 
[12] Viola P, Jones M.: Rapid object detection using a boosted cascade of simple features. In: Computer Vision and Pattern Recognition (CVPR 2001). Proceedings of the 2001 IEEE Computer Society

Conference, Vol. 1; (2001).

[13] Alankar, B., Yousf, N. and Ahsaan, S.U., 2020. Predictive Analytics for Weather Forecasting Using Back Propagation and Resilient Back Propagation Neural Networks. In New Paradigm in Decision Science and Management (pp. 99-115). Springer, Singapore. 